\title{
Left Atrial Appendage Volume and Plasma Docosahexaenoic Acid Levels Are Associated With Atrial Fibrillation Recurrence After Catheter Ablation
}

\author{
Tomoyuki Shiozawa ${ }^{a}$, Kazunori Shimada ${ }^{\text {a d }}$, Gaku Sekita ${ }^{a}$, Hidemori Hayashi ${ }^{a}$, Haruna Tabuchi ${ }^{a}$, \\ Seiji Miura ${ }^{a}$, Shinichiro Fujimoto ${ }^{a}$, Tomoyasu Kadoguchi ${ }^{a}$, Shohei Ouchi ${ }^{\text {a }}$, Tatsuro Aikawa ${ }^{a}$, \\ Hamad Al Shahi ${ }^{a}$, Shuhei Takahashia ${ }^{a}$, Tetsuro Miyazaki ${ }^{b}$, Masataka Sumiyoshic, \\ Yuji Nakazato ${ }^{\mathrm{b}}$, Hiroyuki Daida ${ }^{\mathrm{a}}$
}

\begin{abstract}
Background: Risk factors for atrial fibrillation (AF) recurrence in patients who have undergone AF catheter ablation have not been fully clarified. The objective of this study was to assess whether the left atrium (LA) and LA appendage (LAA) volumes, and cardio-metabolic markers such as polyunsaturated fatty acids (PUFAs) levels were associated with AF recurrence.
\end{abstract}

Methods: Seventy-seven consecutive patients with AF (mean age, 59 \pm 8 years; male, $81 \%$; paroxysmal $\mathrm{AF}, 64 \%$ ) undergoing catheter ablation were enrolled. Using contrast-enhanced cardiac multi-detector computed tomography (MDCT) scan, the LA and LAA volume and orifice area were assessed. Radiofrequency ablation was performed by an irrigation catheter, initially targeting the pulmonary veins with a wide area circumferential ablation.

Results: Patients with AF recurrence (36\%) exhibited both larger LAA volumes and an LAA orifice area than those without AF recurrence, whereas the LA diameter and LA volumes were not significantly different. Notably, AF recurrence occurred in all patients with a large LAA ( $\geq 25 \mathrm{~mL}$ ), and the LAA volume was significantly and negatively associated with docosahexaenoic acid (DHA) levels ( $\beta$ $=-0.33, \mathrm{P}=0.003)$. A multiple regression analysis revealed that the $\log \mathrm{N}$-terminal proB-type natriuretic peptide and plasma DHA levels were independent factors for the LAA volume when adjusted for age, AF detected age, left ventricular (LV) ejection fraction, end-systolic LV diameter.

Manuscript submitted May 4, 2017, accepted June 19, 2017

aDepartment of Cardiovascular Medicine, Juntendo University Graduate School of Medicine, Tokyo, Japan

bDepartment of Cardiology, Juntendo University Urayasu Hospital, Chiba, Japan

'Department of Cardiology, Juntendo University Nerima Hospital, Tokyo, Japan

${ }^{\mathrm{d} C o r r e s p o n d i n g ~ A u t h o r: ~ K a z u n o r i ~ S h i m a d a, ~ D e p a r t m e n t ~ o f ~ C a r d i o v a s c u l a r ~}$ Medicine, Juntendo University Graduate School of Medicine, 2-1-1 Hongo, Bunkyo-ku, Tokyo 113-8421, Japan. Email: shimakaz@juntendo.ac.jp

doi: https://doi.org/10.14740/cr542w
Conclusions: These results suggest that the association between LAA volume and low plasma DHA levels may be an important factor for post-ablation AF recurrence.

Keywords: Atrial fibrillation; Left atrial appendage; Catheter ablation; Docosahexaenoic acid

\section{Introduction}

Catheter ablation for atrial fibrillation (AF) is an established therapy for symptomatic patients with drug refractory AF [1]. Prior to catheter ablation, a multi-detector computed tomography (MDCT) for use with three-dimensional (3D) mapping systems and evaluating the morphology of the pulmonary veins are often performed systematically $[2,3]$. Previous studies have reported several predictors for AF recurrence following catheter ablation [4]. However, there are few studies which have evaluated the association between AF recurrence and cardiac morphology using MDCT. Previous studies have also demonstrated the beneficial effect of omega-3 polyunsaturated fatty acids (PUFAs), including eicosapentaenoic acid (EPA) and docosahexaenoic acid (DHA) for preventing cardiovascular events [5-8]; however, there are only a few reports that have assessed the association between PUFA levels and AF recurrence. Therefore, we assessed the impact of cardiac morphology including left atrial volume and left atrial appendage (LAA) volume, and cardio-metabolic markers such as plasma PUFA levels on AF recurrence in patients undergoing AF catheter ablation.

\section{Materials and Methods}

\section{Subjects}

Seventy-seven consecutive patients (mean age, $59 \pm 8$ years; male, $81 \%$ ) who underwent catheter ablation for symptomatic drug-refractory AF from March 2014 to March 2016 were 
enrolled in this study. The study protocol was approved by the Institutional Review Board of Juntendo University (January 28, 2014, 13-156), and all participants provided written informed consent before their participation in this study according to the guidelines established in the Declaration of Helsinki.

\section{Measurements}

Fasting blood samples were obtained from all patients on the day of the catheter ablation. Serum levels of lipid profiles, high-sensitive $\mathrm{C}$-reactive protein (hsCRP), and $\mathrm{N}$-terminal proB-type natriuretic peptide (NT-proBNP) were measured. Plasma fatty acid levels were measured by 4-fractionation of non-esterified fatty acids by a central laboratory (SRL, Inc., Tokyo, Japan).

Two-dimensional echocardiographic images of the left atrium (LA) and left ventricle (LV) were obtained by the parasternal long axis and apical two- and four-chamber views with second harmonic imaging. Transesophageal echocardiography (TEE) was performed to exclude any atrial thrombi, and to measure the peak velocities of flow out of the left atrial appendage (LAAFV). The standard echocardiographic parameters were obtained on the basis of the American Society of Echocardiography guidelines [9].

All patients underwent a contrast-enhanced cardiac MDCT scan before the catheter ablation procedure for the CARTO mapping system (Biosense Webster, Diamond Bar, CA, USA), using Aquilion ONE ViSION Edition ${ }^{\text {TM }}$ (320-multidetector CT, Toshiba Medical Systems Corporation, Otawara, Japan) and Dual Shot GX 7 (contrast injector, Nemoto Kyorindo Co., Ltd, Tokyo, Japan) with a Model 7800 ECG monitor (Chronos Medical Devices Inc., Tokyo, Japan). Scanning was performed with a slice width of $0.5 \mathrm{~mm}$ and reconstruction interval of $0.25 \mathrm{~mm}$. The prospective computerized tomography angiography (CTA) mode was used for all patients, with a range of X-ray exposure of $35 \%$ of the RR interval. In addition, a 3D workstation (ZIOSTATION2, Ziosoft, Tokyo, Japan) was used to generate 3D structures of the LA and LAA, using the volume-rendered post-processing technique. Standard measurements of the LA, LAA volume, the length of the LAA main lobe, and the LAA orifice were obtained. The LAA orifice cross-sectional area (CSA) was measured manually on multiplanar reformatted images, and its size was defined by its narrowest portion. The formula $0.785 \times$ LAA long diameter $\times$ LAA short diameter was used to calculate a surrogate value for the area of the LAA orifice in accordance with its elliptical shape. Morphology of the LAA was also evaluated using multiplanar reconstruction. LAA morphologies were classified by two cardiologists according to the method provided by Di Biase et al, [10].

\section{Catheter ablation procedure}

All patients received direct oral anticoagulation agents (DOAC), or warfarin with a target international normalized ratio (INR), maintained for at least 3 weeks before the AF catheter ablation. Ablation was performed with the use of radiofrequency energy delivered by a catheter with an open, irrigated tip (Thermocool Smarttouch; Biosense Webster), initially targeting the PVs with a wide area circumferential ablation [11]. After a trans-septal catheterization, and selective PV angiography, extensive encircling pulmonary vein isolation (PVI) was performed [12], using the power control mode with a target power output of $20-35 \mathrm{~W}$, at a maximum temperature of $42{ }^{\circ} \mathrm{C}$, and guided by a 3D electro-anatomical mapping system (CARTO3; Biosense Webster). Intravenous heparin was administered to maintain an activated clotting time of at least 300 - $350 \mathrm{~s}$ during the procedure following the trans-septal catheterization [13]. All cases succeeded in the creation of an extensive ipsilateral bidirectional conduction block between the atrium and PVs. The exit block was examined by pacing from the distal PVs. Adenosine was used as a provocative agent to facilitate PV reconnection [14]. The complete electrical isolation of the PVs was confirmed at least $30 \mathrm{~min}$ after the successful PVI and the restoration of sinus rhythm. If AF was sustained or was induced with a coronary sinus burst pacing at a cycle length down to $200 \mathrm{~ms}$, an additional ablation was performed in second session cases for non-PV foci. This consisted of a linear ablation of the left atrial roof and/or superior vena cava isolation. If the AF did not terminate after an additional ablation, sinus rhythm was restored by an intracardiac cardioversion from the coronary sinus using an atrial cardioversion system (BeeAT; Japan Lifeline, Tokyo, Japan). A cavotricuspid isthmus ablation line was also created for patients with atrial flutter observed during and/or before the ablation, with confirmation of a bidirectional conduction block.

\section{Follow-up}

Following discharge, the patients were followed for 2 - 4 weeks after the catheter ablation and then every $1-3$ months at the outpatient clinic. At each hospital visit, the patients underwent a 12-lead electrocardiogram (ECG) and intensive questioning regarding any arrhythmia-related symptoms. Additionally, $24 \mathrm{~h}$ Holter monitoring was performed at 1, 3, 6, and 12 months following the PVI. Moreover, 12-lead ECGs and $24 \mathrm{~h}$ Holter ECGs were also used anytime the patients reported palpitations. If the ECG revealed any episodes of AF during the follow-up period, the patients were diagnosed with a clinical recurrence of AF irrespective of the presence of symptoms. Patients with AF recurrence were treated temporarily with antiarrhythmic drugs. The discontinuation of antiarrhythmic drugs was then attempted for patients in whom recurrent AF resolved after temporary treatment. If the sinus rhythm could be maintained, these drugs were discontinued permanently. AF was classified as a paroxysmal AF that selfterminates within 7 days, and persistent AF was classified as that which lasts longer than 7 days or requires termination by cardioversion with drugs or by direct current cardioversion and was classified as longstanding persistent AF when AF continued for more than 1 year. Early recurrence of AF was defined as a recurrence of AF within 3 months of ablation, and was considered transient $[1,15]$. AF recurrence was 
Table 1. Clinical Characteristics in Patients With and Without AF Recurrence

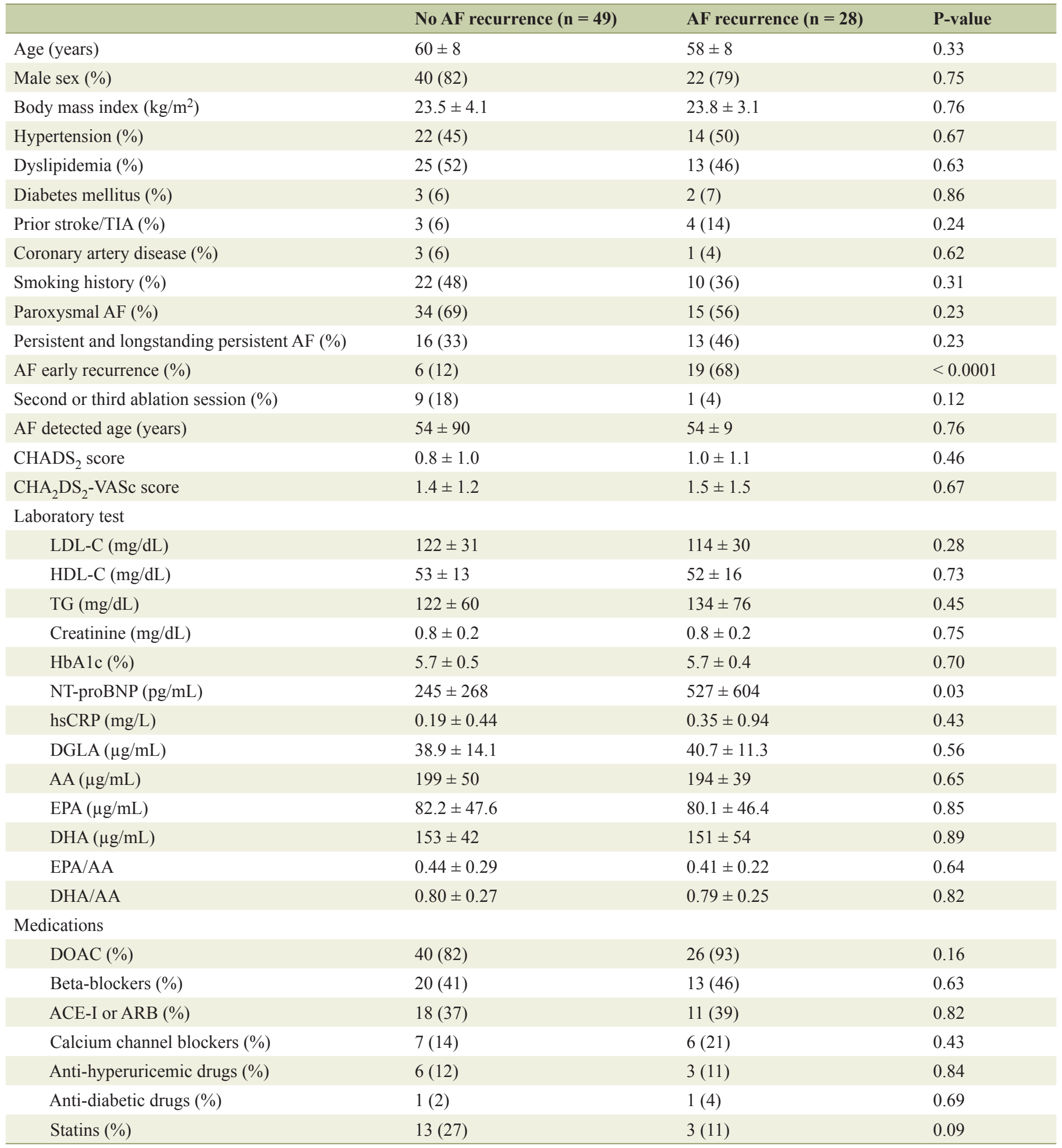

AF: atrial fibrillation; TIA: transient ischemic attack; CHADS $_{2}$ score: congestive heart failure, hypertension, age 75 years or older, diabetes mellitus, and previous stroke or transient ischemic attack; $\mathrm{CHA}_{2} \mathrm{DS}_{2}-\mathrm{VASc}$ score: congestive heart failure, hypertension, age 75 years or older, diabetes mellitus, and previous stroke or transient ischemic attack, vascular disease (coronary artery disease), age 65 - 74 years, and female sex; LDLC: low-density lipoprotein cholesterol; HDL-C: high-density lipoprotein cholesterol; TG: triglycerides; NT-proBNP: N-terminal proB-type natriuretic peptide; hsCRP: high-sensitive C-reactive protein; DGLA: dihomo-gamma-linolenic acid; AA: arachidonic acid; EPA: eicosapentaenoic acid; DHA: docosahexaenoic acid; DOAC: direct oral anticoagulants; ACE-I: angiotensin-converting enzyme inhibitor; ARB: angiotensin-II receptor blocker. 
Table 2. Parameters From Echocardiography and MDCT in Patients With and Without AF Recurrence

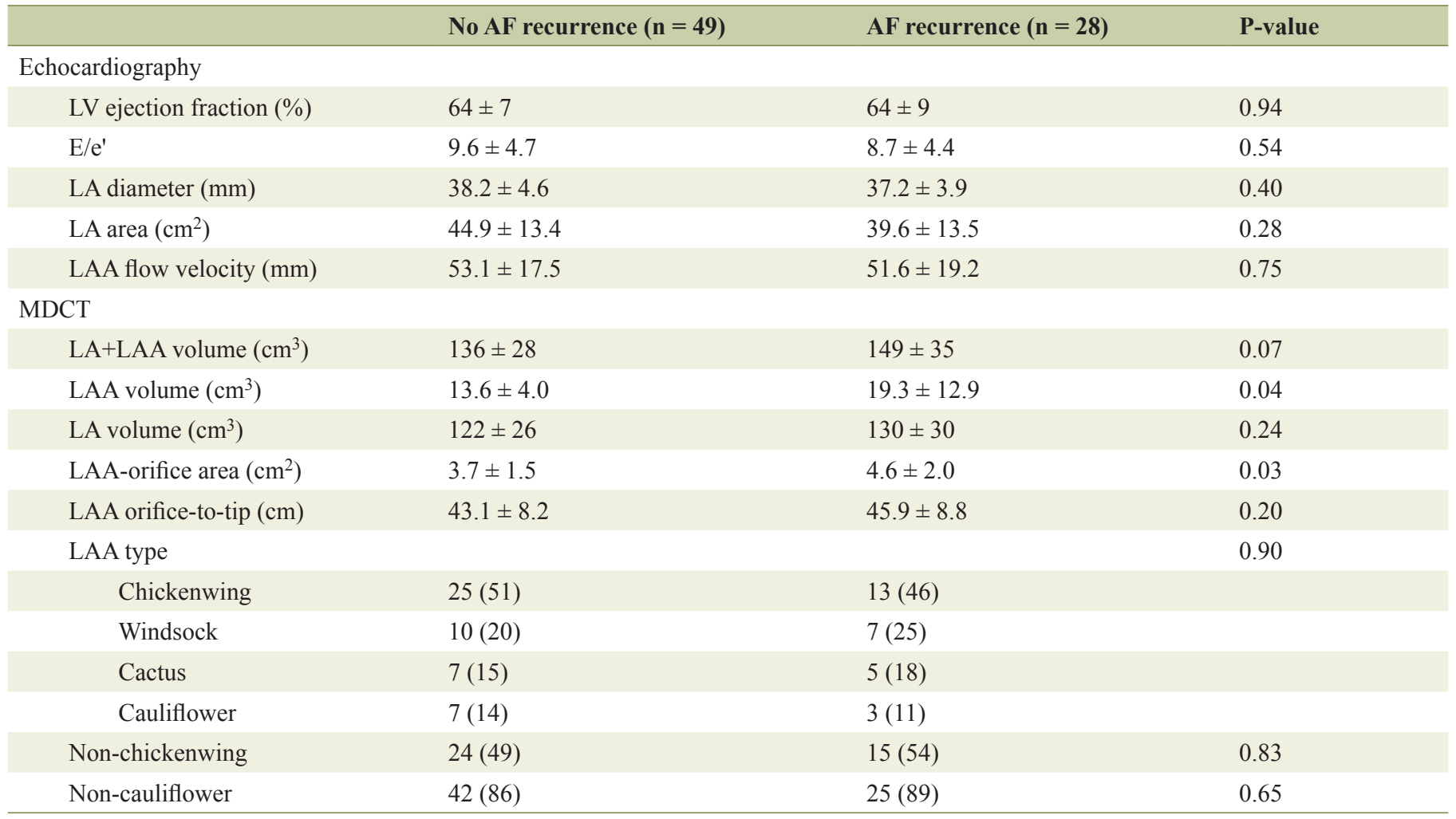

LV: left ventricular ejection fraction; E/e': mitral inflow E/e' ratio determined by Doppler echocardiogram is an indicator of left ventricular diastolic function; LA: left atrium; LAA: left atrium appendage.

defined as the recurrence of AF episodes or any other atrial tachyarrhythmias lasting more than $30 \mathrm{~s}$, after the 3 months blanking period [1].

\section{Statistical analysis}

Data are expressed as the mean \pm standard deviation, or as numbers and percentages. The comparisons between the two groups were tested by an unpaired $t$-test or a MannWhitney $U$ test according to the normality of the data distribution. All of the categorical variables were compared by a Chi-square test or Fisher exact test to detect any differences. The correlations were analyzed using a Spearman's correlation coefficient test. A Cox's proportional hazards model was used to assess the variables independently associated with AF recurrence. Variables incorporated into the Cox's proportional hazard model were selected from variables with a P-value of less than 0.1 in the univariate analysis. If possible, continuous variables were employed unless otherwise specified, including the body mass index divided by the median $\left(23.3 \mathrm{~kg} / \mathrm{m}^{2}\right)$ to analyze obesity, and to prevent the loss of information during the regression analysis. A multiple linear regression analysis was used to assess the relationship between the LAA volumes, including age, systolic LV dimension, LV ejection fraction, AF detected age, $\log$ NT-proBNP, and plasma DHA levels. A P-value of less than 0.05 was regarded to be statistically significant. The statistical analysis was performed using JMP 12 software (SAS Institute Inc., Cary, NC, USA).

\section{Results}

All patients received a successful catheter ablation. There were no patients with DHA containing supplements. During the mean follow-up of $580 \pm 255$ days, 28 (36\%) patients experienced an AF recurrence. The baseline clinical, echocardiographic, and MDCT preprocedural characteristics are presented in Tables 1 and 2 . The prevalence of early AF recurrence before 3 months $(\mathrm{P}<0.0001)$ was significantly higher, and the levels of NT-proBNP $(527 \pm 604 \mathrm{pg} / \mathrm{mL}$ vs. $245 \pm 268 \mathrm{pg} / \mathrm{mL}$, respectively; $\mathrm{P}=0.03$ ) were elevated in patients with an $\mathrm{AF}$ recurrence compared to those in patients without AF recurrence. Although the LA diameter, LAA flow velocity, and LA volume exhibited no significant differences in the two groups, a larger LAA volume $\left(19 \pm 13 \mathrm{~cm}^{3}\right.$ vs. $\left.14 \pm 4 \mathrm{~cm}^{3} ; \mathrm{P}=0.04\right)$ and a larger LAA orifice area $\left(4.9 \pm 2.0 \mathrm{~cm}^{2}\right.$ vs. $3.2 \pm 2.1 \mathrm{~cm}^{2} ; \mathrm{P}=$ $0.001)$ were observed in the patients with an AF recurrence. DHA levels were not associated with the AF recurrence for all subjects. DHA levels were sub-analyzed for the patients with and without hypertension, dyslipidemia, or diabetes mellitus. Non-dyslipidemia patients with an AF recurrence exhibited significantly lower plasma DHA levels $(120 \pm 36 \mu \mathrm{g} / \mathrm{mL}$ vs. 
Table 3. Univariate and Multivariate Cox's Hazard Model Analysis for Variables With AF Recurrence

\begin{tabular}{|c|c|c|c|c|}
\hline & \multicolumn{2}{|c|}{ Univariate analysis } & \multicolumn{2}{|c|}{ Multivariate analysis } \\
\hline & $\begin{array}{l}\text { Hazard ratio } \\
(95 \% \text { confidence interval) }\end{array}$ & P-value & $\begin{array}{l}\text { Hazard ratio } \\
(95 \% \text { confidence interval) }\end{array}$ & P-value \\
\hline Male sex & $0.91(0.41-2.58)$ & 0.91 & & \\
\hline BMI above median & $1.47(0.70-3.18)$ & 0.31 & & \\
\hline Diabetes mellitus & $0.85(0.14-2.86)$ & 0.82 & & \\
\hline Prior stroke or TIA & $1.46(0.43-3.78)$ & 0.50 & & \\
\hline Paroxysmal AF & $0.72(0.34-1.56)$ & 0.39 & & \\
\hline AF detected age per 1 year & $0.98(0.94-1.03)$ & 0.42 & & \\
\hline LA diameter (TTE) per $1 \mathrm{~mm}$ & $0.97(0.88-1.07)$ & 0.52 & & \\
\hline LAA flow velocity (TEE) per $1 \mathrm{~mm}$ & $1.00(0.98-1.02)$ & 0.98 & & \\
\hline LAA volume $(\mathrm{CT})$ per $1 \mathrm{~cm}^{3}$ & $1.04(1.00-1.07)$ & 0.04 & $1.03(1.00-1.06)$ & 0.04 \\
\hline LA volume $(\mathrm{CT})$ per $1 \mathrm{~cm}^{3}$ & $1.01(0.99-1.02)$ & 0.28 & & \\
\hline
\end{tabular}

AF: atrial fibrillation; BMI: body mass index; TIA: transient ischemic attack; NT-proBNP: N-terminal proB-type natriuretic peptide; DHA: docosahexaenoic acid; LA: left atrium; TTE: transthoracic echocardiography; LAA: left atrium appendage; TEE: transesophageal echocardiography; CT: computed tomography.

$148 \pm 37 \mu \mathrm{g} / \mathrm{mL} ; \mathrm{P}=0.03$ ) (Supplementary Figure 1, http:// www.cardiologyres.org). Univariate and multivariate Cox's proportional hazard model analyses are shown in Table 3 . The LAA volume $(\mathrm{P}=0.04)$ was an independent variable for AF recurrence.

The correlation coefficients for the LAA volume are shown in Table 4. The LAA volume was not associated with the type of AF and existence of underlying diseases such as hypertension, diabetes, and dyslipidemia. The LAA volume was significantly and negatively associated with age $(\beta=-0.41, P=0.0003), L V$ ejection fraction $(\beta=-0.37, P=$ $0.005)$, AF detected age $(\beta=-0.32, P=0.008)$, and plasma DHA levels $(\beta=-0.33, P=0.003)$. It was positively associated with the LV diameter at the end systole $(\beta=0.32, \mathrm{P}=$ $0.03)$, and $\log$ NT-proBNP $(\beta=0.31, P=0.01)$. The levels of EPA and arachidonic acid were not associated with the LAA volume. The LAA volume was associated with the LA volume $(\beta=0.35, \mathrm{P}=0.002)$, LAA orifice area $(\beta=0.56, \mathrm{P}$ $<0.0001)$, and LAA length $(\beta=0.48, \mathrm{P}<0.0001)$ measured by MDCT. The correlation between the LAA volume and DHA levels is shown in Figure 1. Notably, AF recurrence occurred in all patients $(n=5)$ with large LAAs $(\geq 25 \mathrm{~mL})$, and these patients had significantly lower levels of DHA than the other patients $(109 \pm 31 \mu \mathrm{g} / \mathrm{mL}$ vs. $157 \pm 46 \mu \mathrm{g} /$ $\mathrm{mL}, \mathrm{P}=0.03)$. In a multivariable regression analysis, $\log$ NT-proBNP $(\beta=0.36, P=0.02)$ and plasma DHA levels $(\beta$ $=-0.39, \mathrm{P}=0.009)$ were independent factors for the LAA volume (Table 4).

\section{Discussion}

\section{Main findings}

This study demonstrates several important findings. First, the LAA volume was an independent factor for AF recurrence following catheter ablation. Second, patients with a large LAA volume, especially an LAA volume $\geq 25 \mathrm{~mL}$ was associated with a high rate of AF recurrence. Third, a large LAA volume was associated with low plasma DHA levels, which are highly distributed within the myocardium.

In this study, we showed that the LAA volume is an independent factor for AF recurrence following ablation. AF is often associated with atrial structural remodeling and chronic inflammation which causes LA fibrosis, scars and dilatation [16]. Previous studies have demonstrated predictors of poor outcomes to be as follows: 1) increased left atrial size; 2) nonparoxysmal AF; 3) sleep apnea and obesity; 4) increased age; 5) hypertension; and 6) left atrial fibrosis detected by a cardiac MRI [4]. Several studies have shown that the LA diameter [17, $18]$ and LA volume $[19,20]$ are predictors of AF recurrence following ablation. In our study, the LAA volume, but not the LA diameter or its volume, was associated with AF recurrence. The patients included in our study were primarily paroxysmal AF or persistent AF with a relatively short duration of AF. It has not been determined whether the increase in LAA volume is a cause or a consequence of AF. The LAA is a remnant of 
Table 4. Univariate and Multivariate Linear Regression Analysis of the Preprocedural Variables Associated With LAA Volume

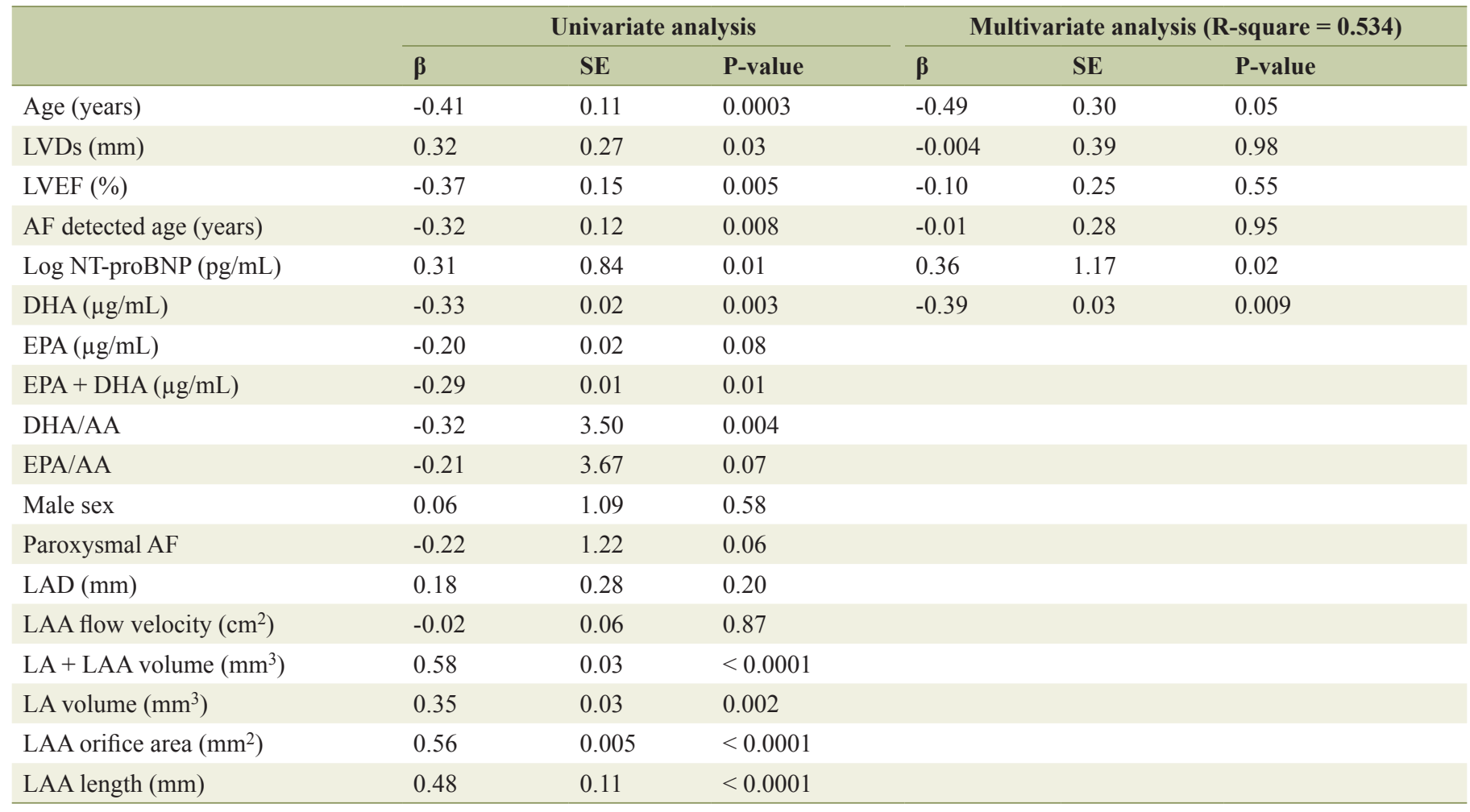

LVDs: systolic left ventricular dimension; LVEF: left ventricular ejection fraction; AF: atrial fibrillation; NT-proBNP: N-terminal proB-type natriuretic peptide; DHA: docosahexaenoic acid; EPA: eicosapentaenoic acid; AA: arachidonic acid; LAD: left atrial dimension; LA: left atrium; LAA: left atrium appendage.

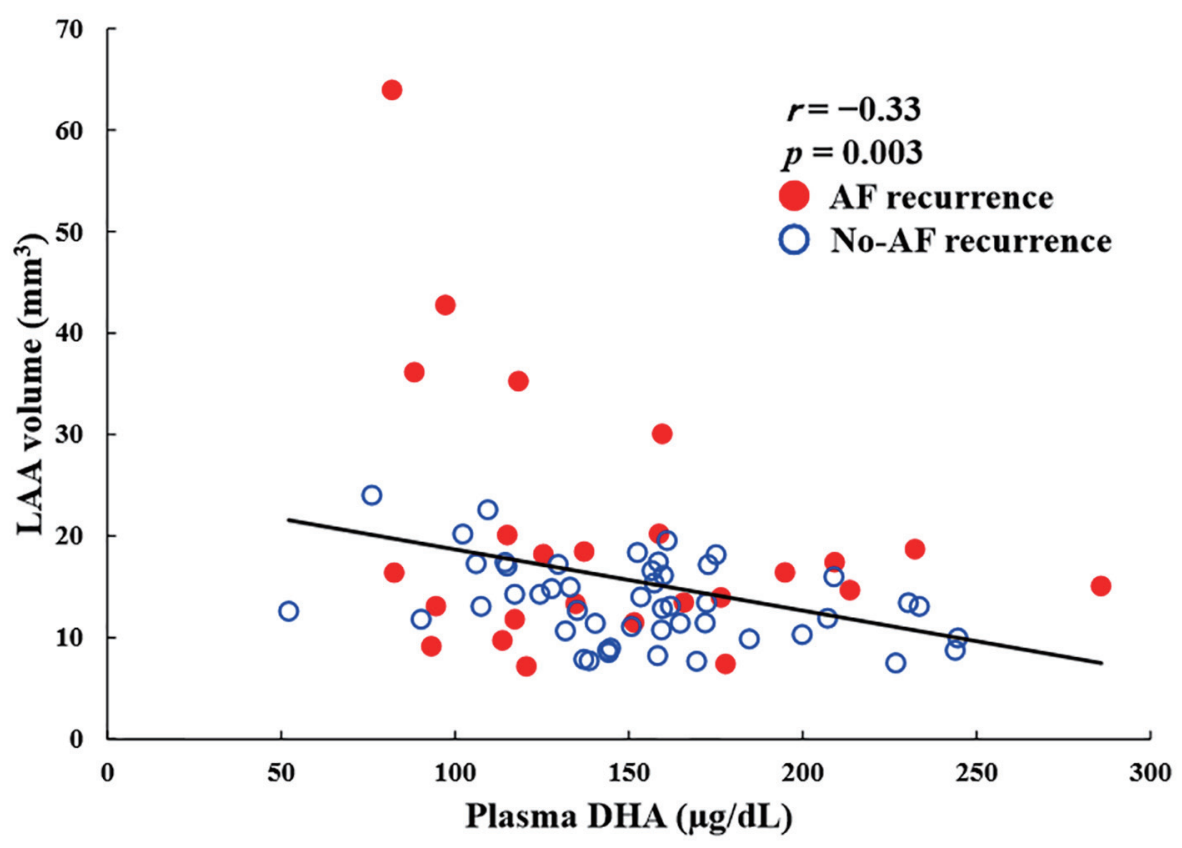

Figure 1. Correlation between plasma DHA levels and LAA volume in AF ablation patients. There was a significant and negative correlation between the two parameters $(r=-0.33, P=0.006)$. Closed circle: AF recurrence group $(n=28)$, open circle: no AF recurrence group $(n=49)$. DHA: docosahexaenoic acid; LAA: left atrial appendage; AF: atrial fibrillation. 
the embryonic left atrium, while the remaining left atrium is derived from an outgrowth of the pulmonary veins [21]. It appears to play an important role in the regulation of heart rate and fluid balance [22] through a distensible reservoir function and its capacity for atrial natriuretic peptide (ANP) secretion, as well as stretch sensitive receptors [23], accompanied by an intrinsic contractile capability. These factors may be associated with the process of atrial remodeling.

\section{NT-proBNP associated with AF recurrence}

Serum NT-proBNP was significantly associated with LAA volume in our study. Elevated natriuretic peptides reflect the myocyte response to increased wall tension, and some studies have indicated that atrial, in addition to ventricular, stretch is a source of BNP [24].

\section{LAA volume in association with AF recurrence}

All patients with a large LAA volume over $25 \mathrm{~mL}$ suffered an AF recurrence. The LAA volume was negatively correlated with age, which may explain why these patients have hereditarily large LAAs. Although the heritability of AF has not fully been identified, AF documentation in a family member is associated with a $40 \%$ increased risk for developing AF $[25,26]$. The LAA is known to be more distensible than the LA, and the LAA volume could be a reliable parameter for determining the LA structural and functional conditions in early AF patients. In this study, the LAA volume was easily measured from an MDCT for the use of CARTO 3D mapping. Therefore, it is important to evaluate the LAA volume to recognize the atrial conditions and predict AF recurrence, especially in patients undergoing a catheter ablation.

\section{DHA levels associated with LAA volume}

In a population-based study in Finland, the serum DHA but not EPA concentration had an impact on AF risk [5], and DHA levels were associated with a lower risk of incident AF, even in the elderly [27]. In the present study, DHA was independently associated with the LAA volume. Previous studies have demonstrated the benefits of omega-3 PUFAs consumption include lowering triglyceride levels, and beneficial effects on vascular and cardiac hemodynamics, including antiarrhythmia and anti-inflammatory responses related to the production of inflammation-resolving mediators [5, 28]. Both EPA and DHA may be associated with health benefits, and the DHA concentration is known to be higher in the human myocardium compared with other organs (e.g., aorta and other vessels). Although our results did not reveal a significant relationship between plasma DHA levels and AF recurrence in all subjects, these may provoke great interest in the mechanisms of DHA consumption that inhibit AF incidence. Patients with non-dyslipidemia and AF recurrence significantly exhibited lower plasma DHA levels, as shown in Figure S1. We believe that confounding factors, such as diet counseling and statin administration, may attenuate associations between plasma DHA levels and AF recurrence. Significant associations between plasma DHA levels and AF recurrence in patients without dyslipidemia may be an important finding in this study. In addition, we should reassess these results with a larger study cohort. Omega-3 PUFAs have both anti-inflammatory and anti-fibrotic properties. These occur through modulation of the phospholipid bilayer and ion channels of the cell membrane as well as by the effect of specialized pro-resolving mediators $[29,30]$. These plasma membrane effects, from DHA, may act on electrophysiological mechanisms. Experimental studies show that DHA, in the myocardium, better protects against ventricular fibrillation than EPA [31]. Few studies have focused on the structural changes resulting from low DHA consumption, and further studies are needed to confirm our finding and to clarify the precise mechanisms by which low DHA levels are associated with structural changes, such as LAA volume enlargement and AF recurrence.

\section{Study limitations}

The present study has several limitations. First, this is a singlecenter study with a relatively small sample size, and a short follow-up duration. Therefore, we included patients with both paroxysmal and persistent AF and some patients undergoing multiple sessions in this study. However, the main results tended to be identical, even in patients with paroxysmal AF and initial ablation procedures (data not shown). Second, the AF recurrence episode was mainly detected by the regular screening during outpatient visits and questioning of symptoms, which may underestimate the true incidence of AF relapse. Third, the LAA and LA volume might be affected by LV diastolic dysfunction, or hemodynamic conditions in patients with AF rhythm. Fourth, we did not evaluate LAA firing in all patients in the present study. It is necessary to investigate the possibility of other AF trigger site in patients with AF recurrence and larger LAA as a next step.

\section{Conclusions}

According to our study, LAA volume was associated with AF recurrence following catheter ablation, especially in patients with large LAAs $(\geq 25 \mathrm{~mL}$ ). Moreover, low levels of DHA were associated with a greater LAA volume. Therefore, the association between the LAA volume and low plasma DHA levels may be an important factor for post-ablation AF recurrence.

\section{Acknowledgments}

The authors would like to thank Shuko Nojiri at the Clinical Research Support Center, Juntendo University for technical advice and support, and Enago (www.enago.jp) and Kostadin Karagiozov for the English language review. 


\section{Grant Support}

This research received no grant from any funding agency in the public, commercial, or not-for-profit sectors.

\section{Financial Disclosure}

K. Shimada received lecture fees from Mochida Pharmaceutical Company and Takeda Pharmaceutical Company. H. Daida received scholarship funds and lecture fees from Mochida Pharmaceutical Company and Takeda Pharmaceutical Company.

\section{Conflicts of Interest}

The other authors report no conflicts of interest.

\section{References}

1. Calkins H, Kuck KH, Cappato R, Brugada J, Camm AJ, Chen SA, Crijns HJ, et al. 2012 HRS/EHRA/ECAS Expert Consensus Statement on Catheter and Surgical Ablation of Atrial Fibrillation: recommendations for patient selection, procedural techniques, patient management and follow-up, definitions, endpoints, and research trial design. Europace. 2012;14(4):528-606.

2. Dong J, Calkins H, Solomon SB, Lai S, Dalal D, Lardo $\mathrm{AC}, \mathrm{Brem} \mathrm{E}$, et al. Integrated electroanatomic mapping with three-dimensional computed tomographic images for real-time guided ablations. Circulation. 2006;113(2):186194.

3. Kanaji Y, Miyazaki S, Iwasawa J, Ichihara N, Takagi T, Kuroi A, Nakamura H, et al. Pre-procedural evaluation of the left atrial anatomy in patients referred for catheter ablation of atrial fibrillation. J Cardiol. 2016;67(1):115-121.

4. Balk EM, Garlitski AC, Alsheikh-Ali AA, Terasawa T, Chung M, Ip S. Predictors of atrial fibrillation recurrence after radiofrequency catheter ablation: a systematic review. J Cardiovasc Electrophysiol. 2010;21(11):12081216.

5. Mozaffarian D, Wu JH. Omega-3 fatty acids and cardiovascular disease: effects on risk factors, molecular pathways, and clinical events. J Am Coll Cardiol. 2011;58(20):2047-2067.

6. Virtanen JK, Mursu J, Voutilainen S, Tuomainen TP. Serum long-chain n-3 polyunsaturated fatty acids and risk of hospital diagnosis of atrial fibrillation in men. Circulation. 2009;120(23):2315-2321.

7. Nishizaki Y, Shimada K, Tani S, Ogawa T, Ando J, Takahashi M, Yamamoto $\mathrm{M}$, et al. Association between the docosahexaenoic acid to arachidonic acid ratio and acute coronary syndrome: a multicenter observational study. BMC Cardiovasc Disord. 2016;16(1):143.

8. Nishizaki Y, Shimada K, Tani S, Ogawa T, Ando J, Takahashi M, Yamamoto M, et al. Significance of imbalance in the ratio of serum n-3 to n-6 polyunsaturated fatty acids in patients with acute coronary syndrome. Am J Cardiol. 2014;113(3):441-445.

9. Zoghbi WA, Enriquez-Sarano M, Foster E, Grayburn PA, Kraft CD, Levine RA, Nihoyannopoulos P, et al. Recommendations for evaluation of the severity of native valvular regurgitation with two-dimensional and Doppler echocardiography. J Am Soc Echocardiogr. 2003;16(7):777-802.

10. Di Biase L, Santangeli P, Anselmino M, Mohanty P, Salvetti I, Gili S, Horton R, et al. Does the left atrial appendage morphology correlate with the risk of stroke in patients with atrial fibrillation? Results from a multicenter study. J Am Coll Cardiol. 2012;60(6):531-538.

11. Haissaguerre M, Jais P, Shah DC, Garrigue S, Takahashi A, Lavergne T, Hocini M, et al. Electrophysiological end point for catheter ablation of atrial fibrillation initiated from multiple pulmonary venous foci. Circulation. 2000;101(12):1409-1417.

12. Ouyang F, Bansch D, Ernst S, Schaumann A, Hachiya H, Chen M, Chun J, et al. Complete isolation of left atrium surrounding the pulmonary veins: new insights from the double-Lasso technique in paroxysmal atrial fibrillation. Circulation. 2004;110(15):2090-2096.

13. Asbach S, Biermann J, Bode C, Faber TS. Early Heparin Administration Reduces Risk for Left Atrial Thrombus Formation during Atrial Fibrillation Ablation Procedures. Cardiol Res Pract. 2011;2011:615087.

14. Hachiya H, Hirao K, Takahashi A, Nagata Y, Suzuki K, Maeda S, Sasaki T, et al. Clinical implications of reconnection between the left atrium and isolated pulmonary veins provoked by adenosine triphosphate after extensive encircling pulmonary vein isolation. J Cardiovasc Electrophysiol. 2007;18(4):392-398.

15. Willems S, Khairy P, Andrade JG, Hoffmann BA, Levesque S, Verma A, Weerasooriya R, et al. Redefining the Blanking Period After Catheter Ablation for Paroxysmal Atrial Fibrillation: Insights From the ADVICE (Adenosine Following Pulmonary Vein Isolation to Target Dormant Conduction Elimination) Trial. Circ Arrhythm Electrophysiol. 2016;9(8): e003909.

16. Letsas KP, Weber R, Burkle G, Mihas CC, Minners J, Kalusche D, Arentz T. Pre-ablative predictors of atrial fibrillation recurrence following pulmonary vein isolation: the potential role of inflammation. Europace. 2009;11(2):158-163.

17. Miyazaki S, Kuwahara T, Kobori A, Takahashi Y, Takei A, Sato A, Isobe M, et al. Preprocedural predictors of atrial fibrillation recurrence following pulmonary vein antrum isolation in patients with paroxysmal atrial fibrillation: long-term follow-up results. J Cardiovasc Electrophysiol. 2011;22(6):621-625.

18. Berruezo A, Tamborero D, Mont L, Benito B, Tolosana JM, Sitges M, Vidal B, et al. Pre-procedural predictors of atrial fibrillation recurrence after circumferential pulmonary vein ablation. Eur Heart J. 2007;28(7):836-841.

19. Abecasis J, Dourado R, Ferreira A, Saraiva C, Cavaco D, Santos KR, Morgado FB, et al. Left atrial volume calculated by multi-detector computed tomography may predict 
successful pulmonary vein isolation in catheter ablation of atrial fibrillation. Europace. 2009;11(10):1289-1294.

20. Helms AS, West JJ, Patel A, Lipinski MJ, Mangrum JM, Mounsey JP, Dimarco JP, et al. Relation of left atrial volume from three-dimensional computed tomography to atrial fibrillation recurrence following ablation. Am J Cardiol. 2009;103(7):989-993.

21. Sherif HM. The developing pulmonary veins and left atrium: implications for ablation strategy for atrial fibrillation. Eur J Cardiothorac Surg. 2013;44(5):792-799.

22. Hoit BD, Shao Y, Gabel M. Influence of acutely altered loading conditions on left atrial appendage flow velocities. J Am Coll Cardiol. 1994;24(4):1117-1123.

23. Al-Saady NM, Obel OA, Camm AJ. Left atrial appendage: structure, function, and role in thromboembolism. Heart. 1999;82(5):547-554.

24. Inoue S, Murakami Y, Sano K, Katoh H, Shimada T. Atrium as a source of brain natriuretic polypeptide in patients with atrial fibrillation. J Card Fail. 2000;6(2):92-96.

25. Lubitz SA, Yin X, Fontes JD, Magnani JW, Rienstra M, Pai M, Villalon ML, et al. Association between familial atrial fibrillation and risk of new-onset atrial fibrillation.
JAMA. 2010;304(20):2263-2269.

26. Tucker NR, Ellinor PT. Emerging directions in the genetics of atrial fibrillation. Circ Res. 2014;114(9):14691482.

27. Wu JH, Lemaitre RN, King IB, Song X, Sacks FM, Rimm EB, Heckbert SR, et al. Association of plasma phospholipid long-chain omega-3 fatty acids with incident atrial fibrillation in older adults: the cardiovascular health study. Circulation. 2012;125(9):1084-1093.

28. Budoff M. Triglycerides and triglyceride-rich lipoproteins in the causal pathway of cardiovascular disease. Am J Cardiol. 2016;118(1):138-145.

29. Harayama T, Eto M, Shindou H, Kita Y, Otsubo E, Hishikawa D, Ishii S, et al. Lysophospholipid acyltransferases mediate phosphatidylcholine diversification to achieve the physical properties required in vivo. Cell Metab. 2014;20(2):295-305.

30. Endo J, Arita M. Cardioprotective mechanism of omega-3 polyunsaturated fatty acids. J Cardiol. 2016;67(1):22-27.

31. McLennan PL. Myocardial membrane fatty acids and the antiarrhythmic actions of dietary fish oil in animal models. Lipids. 2001;36(Suppl):S111-114. 